\title{
Comment améliorer les performances de la recherche clinique en France?
}

\author{
Vincent Diebolt ${ }^{1}$, Yannick Pletan ${ }^{2}$ et les participants à la table ronde $n^{\circ} 4$ de Giens XXIII* \\ 1 GIP CeNGEPS, Direction Générale, Hospices Civils de Lyon, Lyon, France \\ 2 Pfizer, Paris, France
}

Texte reçu le 15 juillet 2008 ; accepté le 30 juillet 2008

\begin{abstract}
Mots clés : recherche clinique; faisabilité ; qualification ; efficacité

Résumé - L'objectif général de l'étude était d'identifier les mesures devant permettre d'améliorer la performance de la recherche clinique en France.

Méthode. Grâce à la discussion entre les membres de la table-ronde (composée de médecins investigateurs ; responsables hospitaliers ; représentants de promoteurs industriels ; médecins généralistes...), nous avons passé en revue les difficultés que rencontrent la recherche clinique française aujourd'hui.

Résultats. Nous avons identifié quatre champs d'action : 1) la disponibilité des ressources nécessaires à la conduite des protocoles de recherche (disponibilité en quantité et en temps, dans les délais les plus brefs); 2) la faisabilité, celle de l'essai dans le design de son protocole, celle du recrutement qui dépend à la fois du centre/équipe/réseau investigateur et des patients et de l'image qu'ils ont de l'expérimentation biomédicale ; 3) la qualification et la motivation des individualités et des métiers associés qui composent l'équipe conduisant les protocoles de recherche ; 4) la fluidité et l'efficacité de l'organisation administrative pour des temps préparatoires au démarrage des essais les plus réduits au profit du temps de réalisation de l'essai.

Conclusion. Cette étude, qui n'a pas la prétention à l'exhaustivité, a montré l'ampleur des efforts pour améliorer l'organisation française d'essais cliniques.
\end{abstract}

D’entrée, il est apparu nécessaire de commencer par préciser le sens donné au terme «performance» dans l'atelier. Une distinction est en effet à opérer entre l'objectif même de la recherche et de ses résultats qui doivent permettre de conclure sur des hypothèses d'ordre scientifique, et sa dimension opérationnelle où il s'agit de satisfaire à un certain nombre de critères internationalement admis, et qui définissent le niveau d'attractivité de la France au sein des pays conduisant ces mêmes activités, dans un contexte international de compétition. C'est à cette seconde dimension, et à elle seule, que s'est intéressée la table ronde.

Dans son déroulement ensuite, la première étape de la réflexion a porté sur l'identification des axes majeurs dans l'amélioration de la recherche clinique. Quatre facteurs ont émergé de cet état des lieux :

\footnotetext{
* Pour la liste des participants, voir en fin d'article.
}

1. La disponibilité des ressources nécessaires à la conduite des protocoles de recherche (disponibilité en quantité et en temps, dans les délais les plus brefs).

2. La faisabilité, celle de l'essai dans le design de son protocole, celle du recrutement qui dépend à la fois du centre/équipe/réseau investigateur (engagement de recrutement) et des patients et de l'image qu'ils ont de l'expérimentation biomédicale.

3. La qualification et la motivation des individualités et des métiers associés qui composent l'équipe conduisant les protocoles de recherche.

4. La fluidité et l'efficacité de l'organisation administrative pour des temps préparatoires au démarrage des essais les plus réduits au profit du temps de réalisation de l'essai.

Pour chacun de ces facteurs qui conditionnent la réalisation dans les délais et les normes de qualités attendues, le groupe de 
réflexion a formulé un certain nombre de recommandations devant permettre d'améliorer la situation actuelle.

\section{Assurer une "opération vérité " sur les ressources (de toutes natures) dédiées et nécessaires}

Aujourd'hui la détermination des moyens nécessaires à la conduite des essais reste artisanale et approximative, sans véritable anticipation de tous les facteurs de production nécessaires. De ce fait, la réalisation de l'essai est handicapée dès son lancement.

Quatre propositions ont été retenues par les participants à la table ronde

1. Développement d'une compétence et d'outils «d'évaluation des moyens nécessaires (temps/coûts) » pour la réalisation des projets cliniques, avec la possibilité de mutualiser les ressources pour les établissements (les centres hospitaliers non universitaires par exemple) n'ayant pas la taille critique ou le niveau d'activité d'essais cliniques en conséquence.

2. Traçabilité et fléchage des financements : reconnaissance aux services opérateurs de ce qui revient à la recherche clinique sans distinction d'origine (que ses ressources soient d'origine publique/PHRC [projet hospitalier de recherche clinique], ou privée/essais à promotion industrielle...).

3. Harmonisation du calcul des surcoûts hospitaliers des essais cliniques avec la définition d'une seule grille en usage dans l'ensemble des établissements hospitaliers. Cette grille globale intégrerait les honoraires versés aux investigateurs (guichet unique pour les industriels) à l'exemple de ce qui existe dans les Centres de lutte contre le cancer.

4. ... et s'assurer que les recettes de la recherche financent bien les activités de recherche...

\section{Garantir une " vraie " faisabilité des essais afin qu'ils soient réalisés en temps voulu avec les chiffres $d$ 'inclusion prévus}

Neuf mesures ont été envisagées par les membres de la table ronde :

1. Assurer la disponibilité d'une compétence logistique professionnelle disponible et dédiée dans les centres d'investigation.

2. Rattachement des personnels de recherche à la Délégation à la recherche clinique et à l'innovation (DRCI), plutôt qu'à des services cliniques, afin d'assurer une mutualisation des moyens et de leur affectation, pour une utilisation optimale par la constitution de pools mobiles.
3. Visibilité de l'offre de service de chaque entité de recherche clinique (hôpital; ville; réseau... ).

4. Recours aux Réseaux. Plusieurs solutions innovantes ont été citées permettant d'activer d'efficaces réseaux « ville hôpital » : solliciter les départements de médecine générale des facultés de médecine; s'appuyer sur les réseaux d'anciens chefs de clinique (CCA) aujourd'hui installés en ville...

5. Information et transparence sur le niveau de performance des centres d'inclusion : mise au point d'un annuaire des centres avec leur orientation scientifique et leur capacité d'inclusion.

6. Prise en compte des différences de cible selon le type d'essai :

- Selon que l'essai porte sur des pathologies chroniques ou rares ou sur des épisodes aigus, les centres d'inclusion à activer ne seront pas les mêmes. Dans le $1^{\text {er }}$ cas, se seront les centres de référence et les associations de patients qu'il conviendra de solliciter; les urgences et les médecins de ville dans le second.

- Faisabilités scientifique et opérationnelle indissociables. Ex. : pour les essais dits en grappe ou en réseau portant sur des maladies rares, le schéma de recrutement est à adapter puisque l'on doit associer le plus grand nombre de centres pour arriver à recruter le nombre attendu de patients qui ne peut qu'être limité. Il y a donc une gestion de la rareté à adapter.

7. Veiller à une plus grande disponibilité des personnels de l'industrie auprès des équipes de réalisation, avec notamment une présence médicale.

8. Développement d'outils d'évaluation des capacités d'inclusion :

- Utilisation du PMSI (Programme de médicalisation des systèmes d'information) et du dossier médical personnel (DMP) lorsqu'il aura été développé.

- Mise en commun des bases de données et registres et cohortes pour identifier les patients « éligibles », dont, pourquoi pas celles dont dispose l'assurance-maladie, en respectant bien entendu l'anonymat des fichiers.

9. Reconnaissance d'un établissement public pharmaceutique national (qui pourrait être l'AGEPS [agence générale des équipements et produits de Santé] à l'Assistance PubliqueHôpitaux de Paris). Il centraliserait la préparation pour les établissements de santé des produits (placebos) et préparations à l'essai thérapeutique ou associés.

\section{Professionnalisation et motivation des personnels : Statut}

\section{1. La formation}

Trois mesures ont été proposées pour valoriser la formation à la recherche clinique. 
- Ouverture sur la recherche clinique dans la formation initiale des différentes professions de santé concernées (lecture critique d'articles [LCA] dans les examens classant nationaux $[\mathrm{ECN}$; ancien internat...]).

- Une prise en compte également en formation continue, diplômante ou validante, bonification. Elle pourrait conduire à la reconnaissance du titre «praticien de recherche clinique » pour le corps médical.

- Diplômante ou validante (Formation médicale continue [FMC]; Évaluation des pratiques professionnelles [EPP]; Validation des acquis [VAE]) : accélération de carrière (médecin, IDE [infirmier diplômé d'état]).

\subsection{Le recrutement}

Quatre actions devraient contribuer à favoriser la professionnalisation de l'activité de recherche clinique.

- Prise en compte de l'expérience et des connaissances en recherche clinique dans les critères/profils de poste des praticiens des établissements de santé.

- Obtention de l'autorisation de lieu des services investigateurs subordonnée à un niveau de formation reconnue des personnels.

- Transparence et structuration de l'intéressement.

- Valorisation de la notion d'équipe dans les publications afin de reconnaître la contribution de tous à la rédaction des articles, et non pas du seul responsable de la structure de recherche.

\subsection{La reconnaissance et la motivation}

Six actions ont été envisagées afin de valoriser la participation à l'activité de recherche clinique.

- Positiver l'image de la recherche clinique [RC] (/personnels +/patients + grand public) : «La RC c'est systématique »... : campagne nationale d'information + actions régionales.

- Présence dans les «steering-committees », ce qui implique une pratique courante de l'anglais.

- Ré-équilibrer la considération du rapport recherche clinique/recherche fondamentale dans la prise en compte de l'évolution de carrière des investigateurs.

- Pérennisation des professionnels dans la recherche clinique à proportion de l'activité « recherche» effective des services.

- Diversification des parcours professionnels accompagné d'une reconnaissance de la formation/spécialisation en recherche clinique (évolution de carrière; bonification indiciaire...).

- Développer les ISS (Investigator Sponsored Studies) : aide supplémentaire au niveau de qualité + accès aux molécules.

\section{Une simplification du processus administratif de mise en place et de conduite des protocoles de recherche}

Des contrats-type en vigueur sur tout le territoire (gain de temps et de personnel).

Un contrat unique pour un établissement donné (plus de convention investigateur individuel associée).

\section{Perspectives}

Les participants de l'atelier ont relevé quelques «bonnes raisons » pour les industries de santé de venir travailler en France :

- Une offre à haute valeur ajoutée pour les essais précoces (phase I, proof of concept, recherche translationnelle) autour de «Centres d'Excellence», avec un personnel très spécialisé, des plateaux techniques et des centres de ressources biologiques accessibles et adaptés. Les «Pôles de Compétitivité » en santé représentent une opportunité majeure, car ils assurent un regroupement territorial et administratif propice.

- Une grande expérience dans les phases tardives de développement, avec une pratique éprouvée et répandue des bonnes pratiques cliniques (BPC) et de l'éthique (historique Loi Huriet). Le développement des réseaux permettant une compétitivité obligatoire sur l'atteinte des objectifs en termes d'effectifs nécessaires, est une étape indispensable à court terme.

- La France est le $3^{\mathrm{e}}$ marché mondial pharmaceutique. Cette position conforte un ensemble de groupes pharmaceutiques internationaux à consentir à des coûts d'étude éventuellement plus élevés pour accéder plus précocement à ce marché. Ceci est particulièrement vrai pour certains domaines thérapeutiques (cardiovasculaires; SNC [système nerveux central] ; rhumatologie)

- Une expertise particulière lui est reconnue dans les domaines du cancer, du SIDA (syndrome d'immunodéficience acquise) et des maladies infectieuses (infections fongiques; antibiothérapie...) expliquant une relative sur représentativité de la France dans ces domaines (30/40\% du développement oncologique mondial sont faits sur le territoire français).

- Ces atouts sont à valoriser au plan international. Pour les phases précoces il s'agit de démontrer la qualité et la facilité de mise en œuvre des meilleures expertises. Pour les phases tardives il faut accepter en revanche d'être comparé sur des « métriques » communément admises au plan international autour des notions de vitesse et de productivité de recrutement, de fiabilité/qualité et de coût/efficience.

- La démarche de valorisation doit être proactive : vis-à-vis des concepteurs d'étude; vis-à-vis des groupes pharmaceutiques, 
En fin d'atelier une liste des 5 actions prioritaires, celles qui ne sauraient attendre, a été dressée... :

- Créer des équipes stables de professionnels au service de l'investigation dans les centres d'inclusion.

- Valoriser l'image de la recherche clinique, de l'expérimentation médicale dans les médias et auprès des patients.

- Harmoniser les pratiques technico-réglementaires.

- Inventer le métier de «logisticien » des essais, celui qui anticipe et assure la résolution des points critiques de faisabilité de l'essai.

- Être pro-actif en sensibilisant les instances et les organismes et en fédérant les initiatives : après du CSIS ; CeNGEPS ; CNCR (qui représentent les 3 conférences) ; Leem; CRBSP; DIRRC/DRCI; INPES...

il s'agit de rencontrer et de convaincre les « développeurs » sur la base de ces critères; de considérer que ces critères évoluent avec le temps et de s'assurer de la persistance de la pertinence de l'offre dans l'environnement concurrentiel (ne pas offrir quelque chose d'obsolète).

\section{Les points non abordés lors de l'atelier}

Certains points n'ont pu être abordés lors de l'atelier, faute de temps. Ils ont pourtant, du point de vue des participants, vocation à être évoqués :

- Rôle du Conseil national de l'ordre des médecins : à sensibiliser (déontologie; aider à la passation de contrats ; vecteur d'image de notoriété).

- Les BioTechs en tant que promoteurs émergents de recherche qui n'ont ni l'expérience, ni les moyens des «big pharmas». Le sujet mérite une réflexion approfondie sur les modèles collaboratifs (partenariats à géométrie variable par exemple dans le cadre des pôles de compétitivité par exemple).

- Les Biobanques; collections qui constituent un élément central des essais de demain (diagnostic, ciblage, réponse thérapeutique, épidémiologie, pharmaco épidémiologie).

- Le rôle des CROs (Contract Research Organisation), comme catalyseurs de performance, et apports de moyens experts (logistiques, compétences, outils, méthodologie...).

- L'AERES (agence d'évaluation de la recherche et de l'enseignement) et son rôle dans la mise au point d'une évaluation performante de la recherche clinique.

- Expérience des SMO (site management organisation) d'utilisation quasi systématique aux États-Unis travaillant pour le compte, soit de sociétés pharmaceutiques, soit de producteurs institutionnels, permettant la mise à disposition locale de personnels et de moyens logistiques facilitant directement l'activité des équipes d'investigation. Le concept de SMO «pures» ne s'est pas imposé en France alors que le besoin est encore plus important, les équipes d'investigation étant proportionnellement plus limitées. De ce fait certaines CROs, qui n'ont pourtant pas le même positionnement ont intégré certaines fonctions logistiques des SMOs.
- Le financement public des projets de recherche clinique (PHRC), qui fertilise le terrain et contribue à la professionnalisation des investigateurs et des équipes de recherche, et dont la pérennité est à assurer.

- Préciput (« over-Head» américain) qui assure une couverture financière complète prenant en compte les frais dits de structure et de management de projets.

- Dépasser le territoire national pour mettre en place un maillage de recherche clinique européenne à partir de la France par la mise en place de réseaux d'investigation identifiés (Ex. à suivre : cardiologie [Lille, Nancy]; SNC/gériatrie [Toulouse]).

\section{Abréviations :}

AERES : Agence d'Évaluation de la Recherche et de l'Enseignement

AGEPS : Agence Générale des Équipements et Produits de Santé - General agency of medical devices and health products (pharmaceutical department of Public Assistance-Hospitals of Paris)

CeNGEPS : Centre national de gestion des essais de produits de santé - National Centre for management of healthcare product trails

CME : Continuing medical education

CNCR : Comité national de coordination de la recherche des CHU - National comity for research activity of the french university hospitals

CNOM : Conseil national de l'Ordre des médecins - French medical National Association

CRBSP : Comité de la Recherche Biomédicale en santé publique - Comity of biomedical research in public health

CRO : Contract Research Organisation

CRP : Clinical research projects

CSIS : Comité Stratégique des industries de santé - Strategic comity of health's industries

DIRRC : Délégation inter-régionale à la recherche clinique Inter-regional delegation of clinical research

DRCI : Délégation à la recherche clinique et à l'innovation Delegation (or Department) of clinical research and innovation 
ECN : Épreuves nationales classantes (ou examen national classant) - National exam for housemen

EPP : Évaluation des pratiques professionnelles - Evaluation of professional practices

INPES : Institut National de Prévention et d'Éducation à la santé

Leem : Les Entreprises du Médicament - Pharma Industry Union

PHRC : Projet hospitalier de recherche clinique

PMSI : Programme de médicalisation des systèmes d'information - Personal medical information system

PMF : Personal medical files

RPL : Recognition of prior learning

SMO : Site management organisation

\section{Participants :}

François Ballet (Sanofi Aventis), Jehan Michel Behier (MSD Chibret), Pierre Bey (Institut Curie, Paris), Antoine Cournot (Théraplix), Hervé Decousus (CHU Hôpital de Bellevue, SaintÉtienne), Jean Deregnaucourt (Pierre Fabre), Élisabeth Devilliers (Fédération Hospitalière de France $[\mathrm{FHF}]$ ), Claude Dubray (CIC, Clermont Ferrand), Claude Gaultier (INSERM), François
Gueyffier (Hôpital Cardiovasculaire Louis Pradel, Lyon Bron), Jean Michel Joubert (UCB Pharma), Rémi Le Coent (GlaxoSmithKline), Françoise Le Thiec (Janssen Cilag), Pascale Maisonneuve (Afssaps), Christine Marey (Science Union Les Laboratoires Servier), Pierre Marquet (Hôpital Dupuytren, Limoges), Dominique Menuet (Pfizer), Emmanuel Ogier (CIC, Brest), Atul Pathak (Pharmacologie Clinique, Toulouse), Éric Postaire (Direction Genérale de la Recherche et de l'Innovation Paris [DGRI]), Olivier Rascol (Faculté de Médecine, Toulouse), Jean Charles Reynier (AP-HM CHU, Marseille), Patrick Rossignol (CIC INSERM, Dommartin les Toul), Christian Thuillez (CHU, Rouen), Françoise Trinquet (AstraZeneca), Philippe Unger (Euraxipharma, Truyes).

Correspondance et offprints : Vincent Diebolt, GIP CeNGEPS, Direction Générale, Hospices Civils de Lyon, 3 quai des Célestins, 69229 Lyon Cedex 02, France.

E-mail : v.diebolt@cengeps.fr 